\title{
Erratum: Nonsteady relaxation and critical exponents at the depinning transition [Phys. Rev. E 87, 032122 (2013)]
}

\author{
E. E. Ferrero, S. Bustingorry, and A. B. Kolton \\ (Received 31 May 2013; published 12 June 2013)
}

DOI: 10.1103/PhysRevE.87.069901

PACS number(s): 64.60.Ht, 75.60.Ch, 05.70.Ln, 99.10.Cd

We found some misprints in our paper. Since some of them are in equations, we believe it is necessary to add this erratum. The conclusions of our work remain unchanged in spite of these corrections.

On p. 2, in the paragraph below Eq. (2), the function $\Delta$ that defines the disorder correlator appears twice as a bivariate function " $\Delta(u, x)$ ". Since our definition of $\Delta$ follows from Eq. (2), $\Delta$ is just a function of $u$. Specifically, $\Delta(x)$ should read $\Delta(u)$ and the integral conditions should read $\int_{u} \Delta(u)=0$ and $\int_{u} \Delta(u)>0$ for the random-bond and random-field cases, respectively.

On p. 3, after Eq. (11), there are minus signs missing in the exponents of the expressions $v \sim \xi^{-\beta / v}$ and $v \sim \ell(t)^{-\beta / v}$.

The sign of the last exponent in Eq. (13) is wrong; it should be positive:

$$
w(t) \sim \ell(t)^{\zeta} \sim t^{\zeta / z}
$$

The same misprint appears after the equation in the text, together with an inexact presentation of the limiting cases for $\tilde{w}$; a reformulated phrase should read as follows: "Otherwise we would have a memory contribution that can be included as $w(t) \sim t^{\zeta / z} \tilde{w}\left(t^{1 / z} L^{-1}\right)$, with $\tilde{w}(y) \sim$ const for $y \lesssim 1$, and a function depending on the initial condition for $y \ll 1$."

Another typo is found on p. 7 in the text after Eq. (17). The correct expression for $w(t)$ is $w(t) \sim t^{\zeta / z}$.

In the caption of Fig. 12, there is a minus sign missing in the time dependence of the velocity, which should be $v \sim t^{-\beta / v z}$. The same mistake appears on p. 10 at the beginning of the first column, where it should read $v=v_{0} t^{-\beta / v z}$.

On p. 11, second column, another exponent sign is missing just before Eq. (25), which should read $f_{c}-f_{c}(L) \sim L^{-1 / v}$.

Equation (25) has missing exponent signs. It should read

$$
v(t) \sim\left[f_{c}-f_{c}(\ell(t))\right]^{\beta} \sim \ell(t)^{-\beta / v} \sim t^{-\beta / v z} .
$$

Later on, in the same column, the previous mistake appears again when presenting the fluctuations of $f_{c}$ in the parabola schema; we should have written $\left[f_{c}-f_{c}(m)\right] \sim L_{m}^{-1 / \nu}$.

Since these errors (mainly missing signs) appear corrected in other parts of the text, we think that the reader will discover them easily. Nevertheless, we want to call special attention to Eqs. (13) and (25). 